\title{
GESTIÓN DE RIESGOS Y ESTRATEGIAS COMUNITARIAS ANTE LOS DESASTRES EN LOCALIDADES DE LA REGIÓN ISTMO-COSTA EN CHIAPAS
}

\section{Risk Management and Community Strategies Following Disasters in Localities of THE IsthMus-CoAst RegION OF CHIAPAS}

\author{
César Aramis Martínez Leina* \\ Guadalupe del Carmen Álvarez Gordillo** \\ Elisa Cruz Rueda***
}

DOI: http://dx.doi.org/10.29043/liminar.v19i2.849

\begin{abstract}
Resumen: En este artículo se exponen aproximaciones al aumento de las condiciones de riesgos presentes en las sociedades, derivado de la progresión de situaciones de vulnerabilidad en los escenarios críticos preexistentes en ellas. En tal sentido, se efectuó un análisis de diagnóstico participativo sobre riesgos y estrategias comunitarias ante los desastres en dos localidades rurales de Pijijiapan, Chiapas. Los resultados muestran la percepción del riesgo, los conflictos sociales que se derivan de estos, así como estrategias de intervención de actores clave y población en general, y también la responsabilidad del Estado para el bienestar y desarrollo con la participación de los niveles institucionales.
\end{abstract}

Palabras claves: gestión de riesgos, desastres naturales, vulnerabilidad, gobernanza, localidades rurales.

Abstract: This article discusses increasing risk conditions in societies, based on growing vulnerability of preexisting perilous scenarios. We undertook a participative diagnostic analysis of risks and community strategies regarding disasters in two rural locations in Pijijiapan, Chiapas. Results demonstrate the perception of risk, social conflicts that derive thereof, intervention strategies of key actors and the general population, and governmental responsibility for overall well-being and development, in tandem with relevant institutions.

Key words: risk management, natural disasters, vulnerability, governance, rural locations.

\footnotetext{
* César Aramis Martínez Leina. Doctor en Estudios Regionales por la Universidad Autónoma de Chiapas, México. Profesor-investigador en la Universidad Autónoma de Chiapas, México. Temas de especialización: política, gestión y desarrollo sostenible. Correo electrónico: cesar. leina@unach.mx. ORCID: https://orcid.org/0000-0002-9460-7764

** Guadalupe del Carmen Álvarez Gordillo. Doctora en Ciencias Biológicas y de la Salud por la Universidad Autónoma Metropolitana, México. Investigadora de El Colegio de la Frontera Sur, México. Temas de especialización: enfermedades consideradas problemas de salud pública, adherencia al tratamiento de enfermedades crónicas, gestión del riesgo
}

de desastres. Correo electrónico: galvarez@ecosur.mx. ORCID: https:// orcid.org/0000-0002-9543-7920.

*** Elisa Cruz Rueda. Doctora en Ciencias Antropológicas por la Universidad Autónoma Metropolitana Unidad Iztapalapa, México. Profesora-Investigadora de la Universidad Autónoma de Chiapas, México. Temas de especialización: derechos humanos, pueblos indígenas y políticas públicas en y para la diversidad. Correo electrónico: elisacruzrueda@hotmail.com. ORCID: https://orcid.org/0000-0002-6339-1518

Enviado a dictamen: 4 de junio de 2020

Aceptación: 2 de febrero de 2021 


\section{Introducción}

L os desastres son la ocurrencia de uno o más agentes perturbadores ${ }^{1}$ de origen natural y socio-organizativo en un determinado territorio (CENAPRED, 2001), que afectan a las poblaciones humanas y su entorno. Por lo general, los desastres ponen en evidencia la desestabilidad en la estructura y el desarrollo de las sociedades, lo que demanda nuevas articulaciones políticas que favorezcan a las poblaciones y gobiernos que se ven afectados por igual, pues se ven en la necesidad de absorber daños que exceden la capacidad de respuesta.

Las catástrofes a nivel mundial han experimentado un incremento en su frecuencia e impacto en los medios de vida de las poblaciones. El cambio climático relacionado con la acción humana en el desarrollo ha incrementado los desastres por fenómenos hidrometeorológicos, la inseguridad alimentaria y los daños a la salud. En México, el sureste es la región más afectada por desastres, ello debido principalmente a la alta vulnerabilidad social, relacionada con la pobreza y la marginación. Las respuestas hasta ahora se han centrado en la mitigación de los daños, pero las amenazas son cada vez mayores y las condiciones de vida de las poblaciones no mejoran, de ahí la importancia de emprender procesos de Gestión del Riesgo de Desastres (GRD).

En México, la política pública de protección civil manifiesta ser el eje rector para la atención de las condiciones de riesgos y desastres de cara a resguardar la seguridad de la población humana, con el diseño, la aplicación, el seguimiento y la evaluación de proyectos y planes de atención en los diferentes niveles de gobierno. Lo anterior tiene sustento en políticas internacionales planeadas en el Marco de Sendai para la reducción de riesgos de desastres (2015-2030), documento adoptado por la Asamblea General de las Naciones Unidas en el que se analizó el esquema de las tendencias de logros y retos de las políticas públicas en protección civil aplicadas en cada Estado-nación (UNISDR, 2015), y en el que se establecieron como prioridades los siguientes puntos: 1) comprender el riesgo de desastre, 2) fortalecer la gobernanza del riesgo de desastres para gestionar dicho riesgo, 3) invertir en la reducción del riesgo de desastres para la resiliencia y 4) aumentar la preparación para casos de desastre a fin de dar una respuesta eficaz y "reconstruir mejor" en los ámbitos de la recuperación, la rehabilitación y la reconstrucción. La GRD es un proceso social, histórico, político y cultural que tiene como finalidad la prevención a largo plazo de los desastres; ello implica la participación de instancias de todos los niveles sociales, internacionales, nacionales, estatales, comunitarios e individuales (Lavell et al., 2003), con principios de sustentabilidad en definición y modos de vida de los mismos involucrados.

Con este concepto se pretende integrar la definición de desastres como construcciones sociales e históricas cuyo resultado es un daño que requiere de ayuda externa ante la imposibilidad de hacerle frente a nivel local. Para la comprensión de los desastres y del riesgo que conllevan, generalmente se desglosan los conceptos de amenaza y vulnerabilidad. El primero se entiende como el agente externo latente que tiene la probabilidad de producir un daño, como por ejemplo inundaciones o sismos.

La identificación de fenómenos naturales que pueden afectar la estructura social obliga a los habitantes a prepararse para atender un posible desastre; a partir del análisis del fenómeno y de las condiciones de riesgo presentes en la zona de afectación, esta identificación representa una orientación cuantitativa y cualitativa para la GRD. El estudio previo permite el diseño de proyectos que integren planes de atención en las fases de intervención; antes, durante y después de la emergencia. La complicación de condicionantes que propician la progresión del riesgo en las sociedades permite estimar la ocurrencia del desastre y el nivel de afectación (Blaikie et al., 1996).

Aunque actualmente se piensa que las amenazas no son totalmente ajenas a la acción humana, el concepto de vulnerabilidad y su atención demandan implementar acciones vinculadas con la Gestión Integral del Riesgo de Desastres (GIRD) que atiendan el supuesto de afectación económica, política o social (Cardona, 2001).

El riesgo es concebido como el resultado de construcciones sociales que están determinadas por las 
fuerzas estructurales de la sociedad y su ambiente (Alcántara-Ayala et al., 2019). La teoría cultural del riesgo subraya el carácter contextual de todas las consideraciones sociales del riesgo y dirige su atención al estudio de la relación entre los distintos marcos de valoración identificables en una sociedad y los riesgos considerados como relevantes en esta (Conejero, 2013).

Por tanto, siendo los desastres resultado de condiciones críticas preexistentes y de vulnerabilidad acumulada, este estudio se centra en las prioridades 1 y 2 de la política pública de protección civil mencionada anteriormente; para tratar la primera de estas prioridades nos interesa el análisis de las percepciones del riesgo, las problemáticas objetivas locales y los conflictos sociales, así como las estrategias que han resultados en procesos de gestión del riesgo eficaces en los contextos comunitarios de la región Istmo-Costa en Chiapas, y también aquellos que han limitado la participación y la sustentabilidad de esas mismas localidades. En cuanto a la segunda prioridad, entendemos que existe una relación estrecha entre gobernanza (acción gobiernosociedad) y gestión de riesgos (acción gubernamental en protección civil o acción ciudadana sin gobierno), porque en ambos se involucra a los actores, su agencia y acción para el manejo del riesgo. De esta manera, la gobernanza examina los espacios en donde interactúan los actores sociales involucrados en la construcción social del riesgo y en su manejo, así como la atención para la mitigación de este, existiendo una corresponsabilidad de autoridades y ciudadanos en la resolución de problemas ya que, como apunta Estrada:

La gobernanza es, en contexto, simultáneamente un resultado de la creciente debilidad del Estado y del gobierno y de su incapacidad para garantizar la gobernabilidad y del fortalecimiento de las formas tradicionales de organización de los actores en las periferias o márgenes, donde por necesidad y por costumbre, predominan las lógicas de los locales y no las del centro, donde predomina el México profundo y no el México imaginario usando la expresión de Bonfil Batalla (Estrada, 2017:84).
Las autoridades reconocen, pues, que forman parte de un sistema que durante mucho tiempo ignoró las capacidades de la ciudadanía y, por tanto, la interlocución con ella, y que por este motivo la ciudadanía no confía en las acciones gubernamentales. A su vez la ciudadanía, conocedora de esto, genera capacidades desde sus espacios y territorios locales, reconociendo que es necesario que el gobierno y sus funcionarios se involucren para hacer cumplir las reglas y el Estado de derecho.

\section{Región de estudio}

La región Istmo-Costa del estado de Chiapas se ha visto afectada históricamente por fenómenos hidrometeorológicos y sismos, los cuales se han agudizado en intensidad y frecuencia en los últimos años. Esta región se localiza al sur sureste de la República Mexicana y al sur del estado de Chiapas, la integra cuatro municipios: Arriaga, Tonalá, Pijijiapan y Mapastepec, en un espacio de 5409.18 kilómetros cuadrados según el marco estadístico del Instituto Nacional de Estadística y Geografía (Gobierno del Estado de Chiapas, 2014). El territorio está integrado por llanuras de distinta altitud, desde 0 hasta 2765 metros sobre el nivel del mar (Martínez, 2017). Se estima una población total de 253427 personas, de las cuales 128059 son mujeres y 125368 hombres. Los municipios de Mapastepec y Pijijiapan, con un total de 111429 habitantes, son considerados como población en condiciones de marginación de riesgo alto; además, el 49.39\% de la población se asienta en localidades consideradas urbanas y el $50.61 \%$ en rurales (Gobierno del Estado de Chiapas, 2020).

La actividad económica principal es la ganadería con los pastizales inducidos y, en menor medida, al natural. También se practica la agricultura de temporal de tomate, sandía, café, mango, palma de aceite y cacao. La pesca es otras de las actividades productivas, es practicada por personas que en su mayoría se sitúan en condiciones de pobreza y que pescan en lagunas costeras, manglares y mar abierto (Martínez, 2017). 


\section{Riesgos de desastres en la región Istmo-Costa}

La presencia de fenómenos geológicos e hidrometeorológicos en la región objeto de estudio pone en condiciones de riesgo alto a las poblaciones, esto debido a la confluencia de tres placas tectónicas: Norteamericana, del Caribe y de Cocos. Dentro de la clasificación de zonas de peligro, la región objeto de estudio se localiza en la zona D, con un nivel del 75\% de gravedad (SGM, 2001). Las variaciones del cambio climático han generado periodos de lluvias con mayor intensidad o bien periodos prolongados de sequía (Medina, 2019).

Se eligieron dos localidades que de manera repetida han presentado inundaciones o sismos en esta región, Ceniceros y El Palmarcito, en el municipio de Pijijiapan, Chiapas. El registro de atención a emergencias por personal operativo de la Secretaría de Protección Civil de la región atiende de manera permanente a 37 localidades con riesgo alto por inundaciones en el municipio de Arriaga, doce para el municipio de Tonalá, cuatro para el municipio de Pijijiapan y once localidades para el municipio de Mapastepec (Martínez, 2017). Sin embargo, de las localidades que se incluyeron en el presente estudio, la primera forma parte de los registros de ayuda en las emergencias de desastres de la Secretaría de Protección Civil de la región, pero actualmente, dada su condición geográfica de planicies y ubicación a los márgenes de los ríos, lagunas con mangle y variaciones en el clima (SGM, 2001), las dos localidades están expuestas a niveles altos de riesgos de origen natural.

Históricamente, las localidades objeto de estudio han sufrido desastres relacionados con fenómenos hidrometeorológicos como el huracán Mitch en 1998 (Rosengaus, Jiménez y Vázquez, 2014) y el huracán Stan en 2005 (CENAPRED, 2006). Respecto a la presencia de fenómenos geológicos, los de mayor afectación fueron el terremoto de 1985, con magnitud de 8.1, y el terremoto con magnitud 8.2 de 2017 (Abeldaño y González, 2018), que dejaron afectaciones principalmente de índole material. Actualmente, a partir de esta memoria, las poblaciones humanas de estas localidades enfrentan emergencias provocadas por fenómenos de estas características, de manera que se organizan por sentido común a nivel familiar y a nivel comunitario para trasladarse a espacios seguros donde resguardar sus vidas e integridad física, así como evitar la pérdida de bienes materiales.

Desde la memoria histórica de los desastres y emergencias presentes se logra identificar las condiciones de riesgo y analizar la vulnerabilidad social construida como producto de procesos de transformación, adaptación y crecimiento de la sociedad, que no han garantizado una adecuada relación entre los ambientes naturales y la construcción del desarrollo sostenible. Estos problemas no resueltos para el progreso (Lavell, 2000) deben integrarse en la GIRD con un enfoque preventivo y con colaboración en dos niveles; el primero es el gubernamental, que comprende lo formal institucional establecido por el Estado, y el segundo nivel es el ciudadano, que incluye la participación de los pobladores y tomadores de decisiones en el entorno local comunitario.

Para la mitigación de los desastres se planea un enfoque preventivo, pero es necesaria una planeación prospectiva por el Estado para la atención de los riesgos y detectar los medios necesarios para alcanzarla; por lo anterior, dicha planeación debe considerar una perspectiva subjetiva que integre la participación de la sociedad, pero visibilizando la diversidad de intereses de los grupos sociales (UNISDR, 2015; Alcántara-Ayala et al., 2019). Así, el Estado tendría que plantearse un trabajo en conjunto sobre la conciencia del riesgo con la participación de todos los actores involucrados, como lo plantea Campos: "las imágenes cognoscitivas que un sujeto concreto, individual o colectivo, desarrolla en relación con las amenazas, con su propia situación de vulnerabilidad y con las relaciones entre ambos aspectos. La conciencia del riesgo no es un reflejo pasivo del riesgo sino su interpretación activa" (Campos, 2005:30).

\section{Metodología}

Esta investigación forma parte del proyecto "Gobernanza y riesgos asociados con los efectos del cambio climático y ambiental en la región Istmo-Costa de Chiapas", financiado por el Programa para el Desarrollo Profesio- 
nal Docente, en su convocatoria 2019. El progreso de la investigación a nivel regional es de tipo polietápico; los resultados que se presentan en este artículo forman parte de la primera etapa en el municipio de Pijijiapan, la cual parte de un modelo para el análisis de los principios de la gestión del riesgo y las propuestas comunitarias (Álvarez, 2011), bajo un enfoque cuali-cuantitativo, ya que incorpora herramientas, instrumentos y técnicas para recolectar información que permita identificar los procesos participativos de la GRD.

Con el objetivo de realizar un diagnóstico participativo sobre riesgos y estrategias comunitarias ante los desastres en las dos localidades rurales mencionadas de Pijijiapan, Chiapas, llevamos a cabo un taller participativo por localidad con actores sociales y población en general, con una duración aproximada de dos horas y 30 minutos, para el que se elaboró una carta descriptiva (ver Tabla l).

La población de estudio, los ejidos Ceniceros y El Palmarcito, se eligió considerando las siguientes variables:

- el alto índice de marginación,

- la ubicación en zonas costeras, por lo que la población ha debido enfrentar situaciones catastróficas ante problemas asociados a eventos hidrometeorológicas y sismos.

Con base en lo anterior, para conocer las percepciones sociales del riesgo, en noviembre y diciembre de 2019 se aplicaron 62 cuestionarios con preguntas semiestructuradas, tomando como unidad de análisis la familia, registrando los datos socioeconómicos, antecedentes de desastres, así como las estrategias de GRD.

\section{Resultados}

\section{Identificación de riesgos en la localidad El Palmarcito}

El ejido El Palmarcito se encuentra aproximadamente a una hora de la ciudad de Pijijiapan. Durante el traslado en auto se pueden apreciar varios poblados, entre ellos uno, llamado Valdivia, que prácticamente desapareció tras las inundaciones provocadas por el huracán Mitch. Después de este encontramos otros donde se reubicaron los sobrevivientes: Milenio Valdivia y Nuevo Valdivia. Pasando estos poblados, y ya en la barra (entre el mar y la ría, entrada del mar o brazo de estero), se encuentra el mar, aproximadamente a 100 o 200 metros; al cruzar el estero por un puente, en menos de diez minutos se encuentra El Palmarcito.

En el taller se contó con la participación del comisariado ejidal, el agente municipal, el comandante, el presidente de la cooperativa de pescadores, el presidente de la cooperativa de palmeros (palma africana), el presidente del comité del Telebachillerato número 71 Francisco Indalecio Madero, el presidente del comité de salud, el consejo de vigilancia de las cooperativas y el consejo de vigilancia del comisariado, con un total de 14 personas.

Se reconoce el medioambiente como un espacio social directamente ligado a las estrategias locales de GRD, como el resultado de la vida cotidiana con implicaciones culturales, mezclado con multiplicidad de actividades y reglas establecidas que permiten el acceso a los recursos y el uso de espacios. Por este motivo, en los talleres resaltamos la valoración del patrimonio biocultural y lo definimos como el conjunto de conocimientos ecológicos locales, culturales y puntos de vista de las personas e instituciones en su interacción con el ambiente. En un ejercicio de mirarnos hacia dentro y valorar estos recursos de vida y ambiente como patrimonio biocultural, los participantes recordaron los momentos que son valiosos para nuestras vidas, en el sentido de trabajar para lo que se consideraba su patrimonio. Los participantes mencionaron que su patrimonio ha sido su propio trabajo, el sustento para la familia, el terreno, la pesca y lo que ofrece el medio de vida.

De las percepciones sobre los desastres, amenazas, vulnerabilidad y riesgos, mediante lluvia de ideas y diálogos con y entre los participantes, se anotaron los principales eventos, conceptos, experiencias y percepciones sobre los conceptos. En general, los desastres se asociaron directamente con el evento de origen natural o social, que son las amenazas, y no con los resultados, con los daños provocados, por la previa vulnerabilidad social 
o su incremento después de los desastres. La vulnerabilidad se asoció principalmente a la falta de acuerdos comunitarios y a conflictos que se desarrollan entre los pobladores y las localidades circunvecinas, relacionados con el acceso a recursos para los medios de vida y con obras de mitigación, así como por las decisiones y acciones llevadas a cabo por los líderes comunitarios y los gobiernos, sin la participación comunitaria principalmente (ver Tabla 2).

Los participantes mencionaron la importancia y la urgencia de realizar acciones para prevenir los desastres. Entre las estrategias, mencionaron que en el pasado con sus recursos comunitarios cerraron la bocabarra a un kilómetro de la boca, en el lado del estero, y dos kilómetros en mar; sin embargo, con el tiempo la necesidad volvió a aparecer por los frecuentes temporales de lluvia. Abordar la problemática central implica que el gobierno realice un estudio y promueva como infraestructura necesaria la construcción de un muelle y de pozos naturales para la obtención de agua dulce, ya que los actuales se han vuelto salados. También mencionaron que los medios de comunicación transmiten mensajes erróneos o que se interpretan mal.

Con relación a la conservación mencionaron los manglares y la necesidad de reforestar en los terrenos de su propiedad, por ejemplo, de 50 a 100 árboles. La mayoría se mostró en disponibilidad de realizar alguna actividad para la comunidad, y mencionaron: "La biblia anuncia la destrucción de la tierra", "Somos de bajos recursos" y "Organizarse, tocar puertas, trabajar".

Durante la reunión se realizaron algunos diálogos sobre las acciones necesarias para la reforestación y la valoración de la participación comunitaria, y no quedarse en la pasividad.

Para identificar objetivamente los riesgos, los participantes hicieron un mapa de la comunidad y señalaron los más importantes; sobre esto, el comisariado dijo: "Estamos rodeados por agua, por tres lados estero, y por el otro el mar". Mediante un mapa explicaron los efectos de la apertura de la bocabarra, que ahora les perjudica porque en el agua de la laguna ya no hay especies de pesca, como el camarón, y el agua potable cada vez es más salobre.
En cuanto a la apertura de la bocabarra todos coincidieron en que se hizo porque se estaba inundando El Palmarcito, aunque algunos discreparon y dijeron que no era necesaria y que se hubiera evitado la tragedia de Valdivia, ya que aunque se inundaran los terrenos de El Palmarcito, no sería la primera vez y el agua no se quedaría estancada, sino que se iría filtrando poco a poco; afirmaron que abrir la bocabarra provocó que al momento de las lluvias el agua proveniente de los cerros aumentara sus corrientes y se disparara con mayor fuerza hacia el mar, lo que afectó a los pobladores de Valdivia. Otro participante mencionó: "Tuvimos que hacerlo, pues de lo contrario nuestros hijos hubieran muerto, y lo que inundó a Valdivia fue el agua que bajó de los cerros, no fue porque se hubiera abierto la bocabarra".

De esta manera, y para trabajar la gobernanza como corresponsabilidades y obligaciones entre el gobierno y la ciudadanía, se les pidió que expusieran sus argumentos imaginándose que uno de nosotros representaba a las instituciones del gobierno, como la Secretaría del Medio Ambiente y Recursos Naturales (SEMARNAT), institución que no quiere concederles el permiso para cerrar la bocabarra, según mencionaron. El ejercicio de diálogo hipotético derivó en que, por un lado, la SEMARNAT señaló: “iPara qué quieren cerrar la bocabarra? ¿Qué garantiza que el recurso federal que se les proporcione no será dinero perdido porque la van a abrir de nuevo?" A lo que ellos respondieron: "No, no lo haremos. Además, nosotros podemos cerrar, no nos des dinero, solo danos el permiso".

\section{Identificación de riesgos en el ejido Ceniceros}

El ejido Ceniceros es una localidad que se encuentra ubicada aproximadamente a 18 kilómetros de la cabecera del municipio de Pijijiapan, Chiapas. La carretera de acceso está pavimentada y pasa por otras comunidades como Guanajuato, Echegaray y Salto de Agua.

Con un total de 25 personas participantes, hombres y mujeres, entre los que se encontraba el agente municipal, se realizó un taller similar al realizado en El Palmarcito en el que se obtuvieron las percepciones sobre desastres, riesgo, vulnerabilidad y GRD. Mediante 
lluvia de ideas se anotaron los principales eventos, conceptos, experiencias y percepciones de estos conceptos (ver Tabla 3).

En la elaboración de un mapa que ejemplificó las situaciones de riesgo, los participantes expusieron la situación de los ríos Coapa Echegaray y Vaca, ya que la localidad se encuentra en medio de los dos ríos y en temporada de lluvia estos salen de su cauce, lo que ocasiona que de manera inmediata, en el transcurso de 30 minutos, la localidad se inunde con corrientes fuertes. Se enfatizó en la necesidad de realizar la compostura de los ríos por el incremento de la frecuencia e intensidad de su curso y del daño que provocan; recientemente se habían producido casos de evacuación de poblaciones y alta contaminación.

Respecto a la gobernanza, se realizó un diálogo entre los participantes para cuestionar el rol del gobierno, así como el costo de las máquinas que se requieren para implementar el desazolve, e incluso se planteó el escenario de que la misma comunidad tuviera el poder para destapar el río; sin embargo, las autoridades locales y los pobladores se mantuvieron en la posición de que la comunidad no cuenta con los insumos para realizar estas acciones.

\section{Percepciones del riesgo de desastres}

En cuanto a las percepciones de los participantes en el taller relacionadas con los desastres, estos no se vislumbran como construcciones sociales, sino que se remiten a la asociación con un evento de origen natural y a la responsabilidad del gobierno para atender las emergencias, principalmente en el caso de El Palmarcito, donde los participantes especificaron tener la necesidad de un plan de atención y de herramientas para hacer frente a los riesgos, en especial al problema de bocabarra que ha provocado salinidad del agua e inundaciones.

Puede afirmarse que lo anterior se debe parcialmente a que los programas gubernamentales no fueron dirigidos a impulsar y fortalecer una gobernanza comunitaria, sino más bien a establecer relaciones de dependencia, de tal forma que ahora consideran que los resultados son responsabilidad del gobierno que los abandonó, y que ellos han perdido la capacidad de organización y de buscar alternativas frente a los cambios en el medioambiente, principalmente el cambio climático.
Entendemos por gobernanza comunitaria [...] aso- ciada a la tradición organizativa de comunidades indígenas y rurales bajo sus formas de democracia directa cuyas decisiones más importantes se toman en asambleas generales y ha sido resultado del entra- mado de relaciones políticas entre comunidades y de estas con el Estado, en este caso sobre todo con los Ayuntamientos (Estrada 2017:43).

De igual forma, los participantes mencionaron que el ser humano no es responsable en su totalidad del cambio climático, el deterioro del suelo, las inundaciones y otros fenómenos que se han clasificado como naturales, por lo que los conceptos de amenazas, vulnerabilidad y riesgo se asumen como los factores que intervienen para que se produzca un desastre, y no se interpretan interrelaciones entre ellos con la participación histórica, la memoria biocultural, las políticas y la acción humana.

Parte de lo anterior propició ciertas tensiones entre los participantes en El Palmarcito por las explicaciones de fenómenos como la inundación de Valdivia o las obras a realizar para atender la bocabarra, de quién es la responsabilidad y cuáles son los caminos para la GRD, incluida la participación de los investigadores.

\section{Percepciones de la población sobre el riesgo de desastres}

Se encuestó a 62 personas, el 48.4\% hombres y el $51.6 \%$, mujeres. El 69.3\% de los encuestados se dedicaban a la pesca, el 9.7\% a la agricultura y el 21\% a otras actividades tales como el comercio, la ganadería y oficios del hogar (ver Tabla 4).

Con base en los eventos de desastres sufridos, la población identificó las inundaciones (71\%) como el riesgo de desastre de mayor afectación, seguido de los sismos y los terremotos; de estos últimos, el de mayor magnitud fue de 8.2 en la escala sismológica de Richter, y fue también el de mayor afectación en la región IstmoCosta de Chiapas en 2017 
A partir de la experiencia vivida por la población ante las emergencias y desastres, el 31\% de los habitantes encuestados considera que el gobierno municipal cuenta con capacidad de respuesta para hacer frente a las emergencias o desastres que puedan afectar a la localidad porque escuchan las propuestas comunitarias, el 68\% considera que no y el 1\% no sabe. La población en general considera que es necesario fortalecer la toma de decisiones, para la ayuda inmediata a las localidades en condiciones de riesgo. Respecto a la gestión local, la población considera que en su mayoría el 45\%, que es de manera regular, el 40\% opina que es una gestión buena, el $7 \%$ que es excelente, el 3\% que es mala, el 3\% que es muy mala y el $2 \%$ no sabe. Los pobladores que contestaron que no se atiende la GIRD desde lo local mencionaron que no habían recibido ningún tipo de ayuda durante las emergencias; también consideraron que el personal operativo no era suficiente, que carecían de equipamiento necesario y que no estaban capacitados para brindar la atención, así mismo, es necesario consolidar los esfuerzos en materia de organización para brindar auxilio a la población durante las emergencias.

Lo anterior refleja que, a partir de la suma de esfuerzos de la organización a nivel local con la gestión de riesgos a nivel municipal, ha permitido resguardar la seguridad de las poblaciones humanas.

\section{Discusión}

En la GRD se involucran tres políticas públicas distintas: la identificación del riesgo - percepción individual, representación social y estimación objetivala reducción del riesgo - prevención, mitigación-y el manejo de desastres - respuesta y recuperaciónTambién es posible analizar y planear la gestión para la reducción o eliminación del riesgo a largo plazo, por lo que las estrategias encaminadas a la prevención implican una identificación objetiva de los riesgos y el trabajo conjunto de los involucrados.

En este sentido, los habitantes de las localidades estudiadas tienen percepciones del riesgo con base en sus experiencias y manifestaron la necesidad de que agentes externos apoyen en la resolución, muchas veces mediante obras de ingeniería, como en el caso de la bocabarra, o mediante proyectos de reforestación o producción a nivel local promovidos por instituciones gubernamentales.

La participación de los investigadores durante los talleres y la aplicación de la encuesta entre la población, primeramente, y los encuentros personales para recabar información sobre los riesgos y los conceptos que se manejan en la localidad, despertaron interés en los participantes en cuanto a la obtención de recursos para emprender obras de mitigación de daños. Durante la exposición de los daños que han sufrido estas poblaciones, pudo observarse cómo se han incrementado las consecuencias de los desastres: el deterioro del suelo, esteros contaminados y poco productivos y, en general, reducción de los medios de producción para la subsistencia de la localidad. Sin embargo, las soluciones propuestas estaban relacionadas en su mayoría con la recepción de recursos económicos para la producción y con servicios de agua, médicos y educativos, así como con obras de mitigación.

Consideramos que los talleres fueron un primer acercamiento para conocer las percepciones del riesgo de desastres, y que comprender las realidades locales implica procesos más amplios y complejos para la gestión del riesgo. Los medios de vida de la población se han deteriorado y se necesitaría evaluar las condiciones de vulnerabilidad social para enfrentar cambios hacia la sustentabilidad.

Por lo anterior, la GIRD es un modelo relativamente nuevo, pues se ha observado que la protección civil y el manejo de riesgos en México y Chiapas no había incidido en un enfoque preventivo a nivel local, de manera que se recurre frecuentemente a la atención posterior a los desastres. Conscientes del avance en el marco normativo, la operación de la ingeniería institucional hace su mayor esfuerzo para la atención a todas las emergencias de riesgos presentes en el municipio y en la región, pero su intervención operativa se hace presente después de la emergencia y no en los procesos de gestión local de riesgos de desastres. 


\section{Conclusiones}

Las localidades estudiadas han padecido desastres y han tenido recientemente experiencias de inundaciones y temblores que se mantienen en la memoria de los pobladores como amenazas latentes, por las que deben elaborarse estrategias de GRD de manera urgente.

Las percepciones del riesgo de desastres entre la población se han construido con base en las experiencias pasadas y en las relaciones que han tenido con las autoridades de los gobiernos local y estatal, además de con representantes institucionales a quienes responsabilizan por las estrategias fallidas hasta ahora.

Se encontraron discursos de colaboración y participación comunitaria para afrontar la GRD, y algunos pobladores han relacionado la acción humana con los resultados derivados del cambio climático, los daños por inundaciones y la baja producción pecuaria. Los participantes en los talleres en El Palmarcito fueron representantes y autoridades reconocidas, y en Ceniceros fueron pobladores, por lo que las estrategias y la definición de los responsables de realizar los cambios en conductas y obras para mitigar daños varían. En cuanto a la responsabilidad del Estado sobre la atención de los derechos a la salud, educación y vivienda, se recomendó identificar las amenazas y vulnerabilidades para promover estrategias de gestión del riesgo, con la participación de todos los niveles institucionales y de la población. Asimismo, se requiere emprender acciones educativas sobre la conciencia del riesgo, dirigidas a personas todas las edades, para alcanzar una participación plena que conduzca a cambios sustentables en los modos de vida y que a largo plazo haga posible prevenir los desastres.

\section{Notas}

${ }^{1}$ Suceso que impacta a un sistema afectable y transformar su estado normal en un estado de daños que llegan al grado de desastres.

\section{Referencias}

Abeldaño Zúñiga, Roberto y Ana María González Villoria (2018). "Desastres en México de 1900 a 2016: patrones de ocurrencia, población afectada y daños económicos". En Revista Panam Salud Pública, 42. Disponible en https://www.scielosp.org/pdf/rpsp/2018. v42/e55 (consultado el 30 de julio de 2020).

Alcántara-Ayala, Irasema et al. (2019). “Gestión Integral de Riesgo de Desastres en México: reflexiones, retos y propuestas de transformación de la política pública desde la academia”. En Investigaciones Geográficas, 98. Disponible en http://www.scielo.org.mx/pdf/ igeo/n98/2448-7279-igeo-98-2.pdf (consultado el 4 de agosto de 2020).

Álvarez Gordillo, Guadalupe del Carmen (2011). Educación y gestión del riesgo de desastres, Procesos educativos en la Cuenca Alta Grijalva. San Cristóbal de Las Casas: El Colegio de la Frontera Sur.

Blaikie, Piers, Terry Cannon Tiers, Ian Davis y Ben Wisner (1996). Vulnerabilidad: el entorno social, político y económico de los desastres. Panamá: Red de Estudios Sociales en Prevención de Desastres en América Latina.

Campos, Armando (2005). Educación y prevención de desastres. S/l: UNICEF, FLACSO, Red de Estudios Sociales en Prevención de Desastres en América Latina. Disponible en https://www.desenredando. org/public/libros/2000/eypd/EducacionYPrevencionDeDesastres-1.0.1.pdf (consultado el 12 de febrero de 2020).

Cardona, Omar Darío (2001). La necesidad de repensar de manera holística los conceptos de vulnerabilidad y riesgo. Una crítica y una revisión necesaria para la gestión. Bogotá: Centro de Estudios sobre Desastres y Riesgos-Universidad de los Andes. Disponible en https://repositorio.gestiondelriesgo.gov.co/ bitstream/handle/20.500.11762/19852/Vulnerab ilidadRiesgoHolistico\%28Cardona_2002\%29. pdf? sequence $=1 \&$ isAllowed $=y$ (consultado el 27 de febrero de 2020).

CENAPRED (Centro Nacional de Prevención de Desastres) (2001). Diagnóstico de peligros e identificación de 
riesgos de desastres en México, Atlas Nacional de Riesgos de la República Mexicana. México: CENAPRED.

CENAPRED (Centro Nacional de Prevención de Desastres) (2006). Características e impacto socioeconómico de los principales desastres ocurridos en la república mexicana en el año 2005. México: CENAPRED. Disponible en http://www.proteccioncivil.gob.mx/work/ models/ProteccionCivil/Resource/375/1/images/ no_7.pdf (consultado el 30 de julio de 2020).

Conejero Paz, Enrique (2013). "Midiendo el riesgo en las administraciones públicas". En Revista de la Facultad de Ciencias Sociales y Jurídicas de Elche, 1(9), 95 113. Disponible en https://www.researchgate.net/ publication/270817656_Midiendo_el_riesgo_en_ las_administraciones_publicas (consultado el 18 de mayo de 2020).

Estrada Guevara (2017). "Gobernanza intercomunitaria del agua para uso doméstico en la región hidropolítica: municipios de Las Margaritas y Maravilla Tenejapa Chiapas". Tesis de Doctorado en Estudios Regionales, Universidad Autónoma de Chiapas, Tuxtla Gutiérrez.

Gobierno del Estado de Chiapas (2014). "Plan estatal de desarrollo Chiapas 2013-2018”. Tuxtla Gutiérrez: Gobierno del Estado de Chiapas. Disponible en http://www.ped.chiapas.gob.mx/ped/wp-content/ uploads/ped/PED_2013-2018_MVC (consultado el 18 de diciembre de 2016).

Gobierno del Estado de Chiapas (2020). Lineamientos para la Programación y Elaboración del Presupuesto de Egresos, Secretaría de Hacienda. Capítulo XXI. Estadística de población. Tuxtla Gutiérrez: Gobierno del Estado de Chiapas. Disponible en http:// haciendachiapas.gob.mx/marco-juridico/Estatal/ informacion/Lineamientos/Normativos/2020/XXVI-Estadistica-Poblacion.pdf (consultado el 22 de mayo de 2020).

Lavell, Alan (2000). "Desastres y desarrollo: hacia un entendimiento de las formas de construcción social de un desastre: el caso del huracán Mitch en Centroamérica". En Garita, Nora y J. Nowalski (comp.), Del desastre al desarrollo sostenible: El caso de Mitch en Centroamérica. S/l: BID, CIDHS. Disponible en https://www.desenredando.org/public/articulos/2000/dyd/DyD2000_mar-1-2002.pdf (consultado el 27 de abril de 2020).

Lavell, Alan et al. (2003). La gestión local de riesgo. Nociones y precisiones en torno al concepto y la práctica. Guatemala: Centro de Coordinación para la Prevención de los Desastres Naturales en América Latina Central-Programa de las Naciones Unidas para el Desarrollo. Disponible en http://www.disaster-info. net/lideres/portugues/brasil\%2006/Material\%20 previo/Allangestriesg.pdf (consultado el 30 de abril de 2020).

Martínez Leina, César Aramis (2017). Gobernanza y gestión de riesgos por inundación: Estudio de caso en los municipios de Tonalá y Pijijiapan pertenecientes a la región Istmo-Costa, Chiapas. Tesis de doctorado en Estudios Regionales, Universidad Autónoma de Chiapas, Tuxtla Gutiérrez.

Medina Sansón, Leopoldo, César Aramis Martínez Leina, Elisa Cruz Rueda, Sylalidia Zárate Jiménez y Paola Chávez Morales (2019). "Problemas relevantes en las respuestas adaptativas sociales y las políticas públicas ante los efectos del cambio climático y ambiental en el estado de Chiapas, México". En Julián Mora Aliseda, Alexandre Castanho y Sergio Neves Lousada Sergio (coords.), Ordenación del espacio: Ciudades inteligentes, Turismo y Logística. Madrid: Aranzadi Thomson Reuters, pp. 409-434.

Rosengaus Moshinsky, Michel, Martín Jiménez Espinosa y María Teresa Vázquez Conde (2014). Atlas climatológico de ciclones tropicales en México. México: Centro Nacional de Prevención de Desastres. Disponible en http://www.cenapred.gob.mx/es/Publicaciones/archivos/37.pdf (consultado el 30 de julio de 2020).

SGM (Servicio Geológico Mexicano) (2001). Atlas de peligros del estado de Chiapas. México: Comité Estatal de Información, Estadística y Geografía de Chiapas. Disponible en http://www.ceieg.chiapas.gob.mx/productos/files/RPSGM/informe_final_peligros_chiapas.pdf (consultado el 4 de mayo de 2020). 
UNISDR (Oficina de las Naciones Unidas para la Reducción del Riesgo de Desastres) (2015). Marco de Sendai para la reducción de riesgo de desastres 2015-2030.
Ginebra: UNISDR. Disponible en https://www. unisdr.org/files/43291_spanishsendaiframeworkfordisasterri.pdf (consultado el 23 de mayo de 2020).

Tabla 1. Objetivos y descripción de temas trabajados con participantes de las localidades estudiadas

\section{Tema}

Bienvenida, introducción, objetivos y resultados esperados.

Lluvia de ideas para conocer sobre las percepciones y significados del desastre: desastre, vulnerabilidad, amenaza.

Experiencias ante el desastre.

¿Cuál es nuestro patrimonio?

¿Cuáles son los riesgos de desastres en mi comunidad?

Las estrategias que hemos utilizado para prevenir y enfrentar desastres.

Conclusiones.

Fuente: elaboración propia.

\section{Producto}

Alcance de objetivos de la reunión.

Percepciones sobre los desastres.

Reconocimiento y conciencia del riesgo.

Introducir en el análisis el concepto de patrimonio biocultural.

Mapa y registro priorizado de riesgos de desastres.

Lluvia de ideas y experiencias.

Retroalimentación de resultados. 
Tabla 2. Percepciones del riesgo y desastres en la localidad El Palmarcito

\section{Los desastres}

Amenaza

Vulnerabilidad

Riesgo

Inundación.

Temblor.

Incendio.

Huracanes.

Temporal de agua.

Tener miedo.

Barbasco con muerte de peces.

Quema de casas.

Basura en localidad.

Tirar botellas en esteros.

Pérdida de patrimonio.

Pérdida de siembras.

Separación de mujer-matrimonio o perder un familiar.

Epidemias.

Gobiernos malos, robar dinero.

Lo que hace el río.

Bocabarra, se lleva piedras el río.

Epidemia en el ganado por rabia.

No tener producción en sistema lacunario, Carretas Pereira, palma de aceite.

Tapar carreteras.
Gobierno y
empresarios
extranjeros,
europeos que
invierten.
No cumplen las
peticiones.
Cinco cooperativas en
la localidad.

Crimen organizado.

Miedo.

Burla.

Dominio de las

comunidades.

Secuestro.

Vandalismo.

Desastre.

Mentir.

Zapatistas.

Los que quemaron

bandera.

Protección civil.

Comités.

Gobierno debe apoyar con herramientas.

Nos urge

organizarnos.

Actuar.

Cumplir.

Exigir.

Presencia de fuereños.

Hambre porque no hay producción.

Salud, dengue por mucho zancudo, no hay médico, ni medicina IMSS.

Ríos fueron canalizados por el gobierno y acabaron el matrimonio.

Nosotros mismos estamos haciendo desastres.

Fuente: elaboración propia. 
Tabla 3. Percepciones del riesgo, desastres y estrategias en el poblado Ceniceros

\begin{tabular}{|c|c|c|c|}
\hline Desastres & Vulnerabilidad & Riesgo & Estrategias \\
\hline $\begin{array}{l}\text { Terremoto. } \\
\text { Temblor. } \\
\text { Inundación por ríos. } \\
\text { Accidentes. } \\
\text { Falta de alimentos. } \\
\text { Lo que hace el rio. } \\
\text { Quema de casas. } \\
\text { Mortalidad. } \\
\text { Hacer negocios. } \\
\text { Daño. } \\
\text { Fuertes lluvias. } \\
\text { Lo que hace la } \\
\text { naturaleza. } \\
\text { Desastre natural y } \\
\text { comunidad que no } \\
\text { sabe hacerles frente. }\end{array}$ & $\begin{array}{l}\text { Inicialmente término } \\
\text { desconocido. } \\
\text { Malestar. } \\
\text { Falta de recursos. } \\
\text { No lo han } \\
\text { escuchado. } \\
\text { Incendio en casa. } \\
\text { Falta de recursos. } \\
\text { Compadecerse de } \\
\text { las personas. }\end{array}$ & $\begin{array}{l}\text { Peligro. } \\
\text { Pérdida y daño. } \\
\text { Inundación que llevó } \\
\text { gallinas y trastes. } \\
\text { La caída de la casa } \\
\text { ejidal. } \\
\text { Inversión en el } \\
\text { cultivo. } \\
\text { Posibilidad o } \\
\text { suposición de que } \\
\text { algo puede pasar. }\end{array}$ & $\begin{array}{l}\text { Compostura de los ríos. } \\
\text { Diseño de mecanismo para colgar } \\
\text { mochilas. } \\
\text { Comunicación con el presidente. } \\
\text { Desazolve de los ríos. } \\
\text { Evacuar con cayuco. } \\
\text { Fortalecer las viviendas que están a } \\
\text { las orillas del río. } \\
\text { Enrocamiento de la orilla del río. } \\
\text { Desazolve del rio con máquinas. } \\
\text { Vigilar el rio cuando esté alto. } \\
\text { La gestión del riesgo pretende que } \\
\text { la comunidad en conjunto pueda } \\
\text { determinar las líneas de acción. } \\
\text { El gobierno debe dar las máquinas y } \\
\text { la gente debe destapar. }\end{array}$ \\
\hline
\end{tabular}

Fuente: elaboración propia.

Tabla 4. Características sociodemográficas de la población

\begin{tabular}{|c|c|c|c|c|c|c|}
\hline \multirow{2}{*}{ Localidades } & \multicolumn{2}{|c|}{ Edades } & \multicolumn{2}{|c|}{ Sexo } & \multicolumn{2}{|c|}{ Ocupación } \\
\hline & Grupo de edades & Número & Hombres & Mujeres & Actividad & Número \\
\hline \multirow[t]{5}{*}{ Ejido El Palmarcito } & 20-30 años & 1 & 17 & 15 & Agricultura & 1 \\
\hline & 31-40 años & 9 & & & & \\
\hline & 41-50 años & 7 & & & Pesca & 27 \\
\hline & 51-60 años & 10 & & & & \\
\hline & 61-70 años & 5 & & & Otra & 4 \\
\hline \multirow[t]{7}{*}{ Ejido Ceniceros } & 18-20 años & 3 & 13 & 17 & Agricultura & 5 \\
\hline & 21-30 años & 5 & & & & \\
\hline & 31-40 años & 5 & & & Pesca & 16 \\
\hline & 41-50 años & 7 & & & & \\
\hline & 51-60 años & 4 & & & & \\
\hline & 61-70 años & 1 & & & Otras & 9 \\
\hline & 71-80 años & 5 & & & & \\
\hline
\end{tabular}

Fuente: elaboración propia. 